\title{
Reducing Concurrent Analysis Under a Context Bound to Sequential Analysis ${ }^{\star}$
}

\author{
Akash Lal ${ }^{1, \star \star}$ and Thomas Reps ${ }^{1,2}$ \\ ${ }^{1}$ University of Wisconsin; Madison, WI; USA \\ \{akash, reps\}@cs.wisc.edu \\ ${ }^{2}$ GrammaTech, Inc., Ithaca, NY, USA
}

\begin{abstract}
This paper addresses the analysis of concurrent programs with shared memory. Such an analysis is undecidable in the presence of multiple procedures. One approach used in recent work obtains decidability by providing only a partial guarantee of correctness: the approach bounds the number of context switches allowed in the concurrent program, and aims to prove safety, or find bugs, under the given bound. In this paper, we show how to obtain simple and efficient algorithms for the analysis of concurrent programs with a context bound. We give a general reduction from a concurrent program $P$, and a given context bound $K$, to a sequential program $P_{s}^{K}$ such that the analysis of $P_{s}^{K}$ can be used to prove properties about $P$. We give instances of the reduction for common program models used in model checking, such as Boolean programs and pushdown systems.
\end{abstract}

\section{Introduction}

The analysis of concurrent programs is a challenging problem. While in general the analysis of both concurrent and sequential programs is undecidable, what makes concurrency hard is the fact that even for simple program models, the presence of concurrency makes their analysis computationally very expensive. When the model of each thread is a finite-state automaton, the analysis of such systems is PSPACE-complete; when the model is a pushdown system, the analysis becomes undecidable [18. This is unfortunate because it does not allow the advancements made on such models in the sequential setting, i.e., when the program has only one thread, to be applied in the presence of concurrency.

This paper addresses the problem of automatically extending analyses for sequential programs to analyses for concurrent programs under a bound on the number of context switches 1 We refer to analysis of concurrent programs under a context bound as context-bounded analysis (CBA). Previous work has shown the value of CBA: KISS [17, a model checker for CBA with a fixed context

\footnotetext{
* Supported by NSF under grants CCF-0540955 and CCF-0524051 and by AFRL under contract FA8750-06-C-0249.

** Supported by a Microsoft Research Fellowship.

${ }^{1}$ A context switch occurs when execution control passes from one thread to another.
}

A. Gupta and S. Malik (Eds.): CAV 2008, LNCS 5123, pp. 37-51, 2008.

(C) Springer-Verlag Berlin Heidelberg 2008 
bound of 2, found numerous bugs in device drivers; a study with explicit-state model checkers [13] found more bugs with slightly higher context bounds. It also showed that the state space covered with each increment to the context-bound decreases as the context bound increases. Thus, even a small context bound is sufficient to cover many program behaviors, and proving safety under a context bound should provide confidence towards the reliability of the program. Unlike the above-mentioned work, this paper addresses CBA with any given context bound and with different program abstractions (for which explicit-state model checkers would not terminate).

The decidability of CBA, when each program thread is abstracted as a pushdown system (PDS) - which serves as a general model for a recursive program with finite-state data - was shown in [16. These results were extended to PDSs with bounded heaps in [3] and to weighted PDSs (WPDSs) in [10]. All of this work required devising new algorithms. Moreover, each of the algorithms have certain disadvantages that make them impractical to implement.

In the sequential setting, model checkers, such as those described in [121.5], use symbolic techniques in the form of BDDs for scalability. With the CBA algorithms of [163, it is not clear if symbolic techniques can be applied. Those algorithms require the enumeration of all reachable states of the shared memory at a context switch. This can potentially be very expensive. However, those algorithms have the nice property that they only consider those states that actually arise during valid (abstract) executions of the model. (We call this lazy exploration of the state space.)

Our recent paper [10] showed how to extend the algorithm of [16] to use symbolic techniques. However, the disadvantage there is that it requires computing auxiliary information for exploring the reachable state space. (We call this $e a$ ger exploration of the state space.) The auxiliary information summarizes the effect of executing a thread from any control location to any other control location. Such summarizations may consider many more program behaviors than can actually occur (whence the term "eager").

This problem can also be illustrated by considering interprocedural analysis of sequential programs: for a procedure, it is possible to construct a summary for the procedure that describes the effect of executing it for any possible inputs to the procedure (eager computation of the summary). It is also possible to construct the summary lazily (also called partial transfer functions [12) by only describing the effect of executing the procedure for input states under which it is called during the analysis of the program. The former (eager) approach has been successfully applied to Boolean programs 1 , but the latter (lazy) approach is often desirable in the presence of more complex abstractions, especially those that contain pointers (based on the intuition that only a few aliasing scenarios occur during abstract execution). The option of switching between eager and lazy exploration exists in the model checkers described in 120 .

${ }^{2}$ Boolean programs are imperative programs with only the Boolean datatype (3). 
Contributions. This paper makes two main contributions. First, we show how to reduce a concurrent program to a sequential one that simulates all its executions for a given number of context switches. This has the following advantages:

- It allows one to obtain algorithms for CBA using different program abstractions. We specialize the reduction to Boolean programs (\$3), PDSs (\$4), and symbolic PDSs (see [8]). The former shows that the use of PDS-based technology, which seemed crucial in previous work, is not necessary: standard interprocedural algorithms [19227] can also be used for CBA. Moreover, it allows one to carry over symbolic techniques designed for sequential programs for CBA.

- The reduction introduces symbolic constants and assume statements. Thus, any sequential analysis that can deal with these two additions can be extended to handle concurrent programs as well (under a context bound).

- For the case in which a PDS is used to model each thread, we obtain better asymptotic complexity than previous algorithms, just by using the standard PDS algorithms (\$4).

- The reduction shows how to obtain algorithms that scale linearly with the number of threads (whereas previous algorithms scaled exponentially).

Second, we show how to obtain a lazy symbolic algorithm for CBA on Boolean programs (\$5). This combines the best of previous algorithms: the algorithms of 16.3 are lazy but not symbolic, and the algorithm of 10 is symbolic but not lazy.

The rest of the paper is organized as follows: 92 gives a general reduction from concurrent to sequential programs; 93 specializes the reduction to Boolean programs; $\$ 4$ specializes the reduction to PDSs; $\$ 5$ gives a lazy symbolic algorithm for CBA on Boolean programs; 6 reports early results with our algorithms; 97 discusses related work. Additional details and proofs can be found in [8].

\section{A General Reduction}

This section gives a general reduction from concurrent programs to sequential programs under a given context bound. This reduction transforms the nondeterminism in control, which arises because of concurrency, to non-determinism on data. (The motivation is that the latter problem is understood much better than the former one.)

The execution of a concurrent program proceeds in a sequence of execution contexts, defined as the time between consecutive context switches during which only a single thread has control. In this paper, we do not consider dynamic creation of threads, and assume that a concurrent program is given as a fixed set of threads, with one thread identified as the starting thread.

Suppose that a program has two threads, $T_{1}$ and $T_{2}$, and that the context bound is $2 K-1$. Then any execution of the program under this bound will have up to $2 K$ execution contexts, with control alternating between the two threads, informally written as $T_{1} ; T_{2} ; T_{1}, \cdots$. Each thread has control for at most 
$K$ execution contexts. Consider three consecutive execution contexts $T_{1} ; T_{2} ; T_{1}$. When $T_{1}$ finishes executing the first of these, it gets swapped out and its local state, say $l$, is stored. Then $T_{2}$ gets to run, and when it is swapped out, $T_{1}$ has to resume execution from $l$ (along with the global store produced by $T_{2}$ ).

The requirement of resuming from the same local state is one difficulty that makes analysis of concurrent programs hard - during the analysis of $T_{2}$, the local state of $T_{1}$ has to be remembered (even though it is unchanging). This forces one to consider the cross product of the local states of the threads, which causes exponential blowup when the local state space is finite, and undecidability when the local state includes a stack. An advantage of introducing a context bound is the reduced complexity with respect to the size $|L|$ of the local state space: the algorithms of 163 scale as $\mathcal{O}\left(|L|^{5}\right)$, and 10 scales as $\mathcal{O}\left(|L|^{K}\right)$. Our algorithm, for PDSs, is $\mathcal{O}(|L|)$. (Strictly speaking, in each of these, $|L|$ is the size of the local transition system.)

The key observation is the following: for analyzing $T_{1} ; T_{2} ; T_{1}$, we modify the threads so that we only have to analyze $T_{1} ; T_{1} ; T_{2}$, which eliminates the requirement of having to drag along the local state of $T_{1}$ during the analysis of $T_{2}$. For this, we assume the effect that $T_{2}$ might have on the shared memory, apply it while $T_{1}$ is executing, and then check our assumption after analyzing $T_{2}$.

Consider the general case when each of the two threads have $K$ execution contexts. We refer to the state of shared memory as the global state. First, we guess $K-1$ (arbitrary) global states, say $s_{1}, s_{2}, \cdots, s_{K-1}$. We run $T_{1}$ so that it starts executing from the initial state $s_{0}$ of the shared memory. At a nondeterministically chosen time, we record the current global state $s_{1}^{\prime}$, change it to $s_{1}$, and resume execution of $T_{1}$. Again, at a non-deterministically chosen time, we record the current global state $s_{2}^{\prime}$, change it to $s_{2}$, and resume execution of $T_{1}$. This continues $K-1$ times. Implicitly, this implies that we assumed that the execution of $T_{2}$ will change the global state from $s_{i}^{\prime}$ to $s_{i}$ in its $i^{\text {th }}$ execution context. Next, we repeat this for $T_{2}$ : we start executing $T_{2}$ from $s_{1}^{\prime}$. At a nondeterministically chosen time, we record the global state $s_{1}^{\prime \prime}$, we change it to $s_{2}^{\prime}$ and repeat $K-1$ times. Finally, we verify our assumption: we check that $s_{i}^{\prime \prime}=s_{i+1}$ for all $i$ between 1 and $K-1$. If these checks pass, we have the guarantee that $T_{2}$ can reach state $s$ if and only if the concurrent program can have the global state $s$ after $K$ execution contexts per thread.

The fact that we do not alternate between $T_{1}$ and $T_{2}$ implies the linear scalability with respect to $|L|$. Because the above process has to be repeated for all valid guesses, our approach scales as $\mathcal{O}\left(|G|^{K}\right)$, where $G$ is the global state space. In general, the exponential complexity with respect to $K$ may not be avoidable because the problem is NP-complete when the input has $K$ written in unary 9 . However, symbolic techniques can be used for a practical implementation.

We show how to reduce the above assume-guarantee process into one of analyzing a sequential program. We add more variables to the program, initialized with symbolic constants, to represent our guesses. The switch from one global state to another is made by switching the set of variables being accessed by the program. We verify the guesses by inserting assume statements at the end. 


\begin{tabular}{|c|c|c|}
\hline Program $P^{s}$ & $\overline{\text { st } \in T_{i}}$ & Checker \\
\hline $\begin{array}{l}L_{1}: T_{1}^{s} ; \\
L_{2}: T_{2}^{s} ; \\
L_{3}: \text { Checker }\end{array}$ & $\begin{array}{l}\text { if } \mathrm{k}=1 \text { then } \\
\tau(\mathrm{st}, 1) ; \\
\text { else if } \mathrm{k}=2 \text { then } \\
\tau(\mathrm{st}, 2) ; \\
\quad \cdots \\
\text { else if } \mathrm{k}=K \text { then } \\
\quad \tau(\mathrm{st}, K) \\
\text { end if } \\
\text { if } \mathrm{k} \leq K \text { and } * \text { then } \\
\mathrm{k}++ \\
\text { end if } \\
\text { if } \mathrm{k}=K+1 \text { then } \\
\mathrm{k}=1 \\
\text { goto } L_{i+1} \\
\text { end if }\end{array}$ & $\begin{array}{l}\text { for } i=1 \text { to } K-1 \text { do } \\
\quad \text { for } j=1 \text { to } n \text { do } \\
\quad \text { assume }\left(\mathrm{x}_{j}^{i}=v_{j}^{i+1}\right) \\
\text { end for } \\
\text { end for }\end{array}$ \\
\hline
\end{tabular}

Fig. 1. The reduction for general concurrent programs under a context bound $2 K-1$. In the second column, * stands for a nondeterministic Boolean value.

The reduction. Consider a concurrent program $P$ with two threads $T_{1}$ and $T_{2}$ that only has scalar variables (i.e., no pointers, arrays, or heap) 3 We assume that the threads share their global variables, i.e., they have the same set of global variables. Let $\mathrm{VAR}_{G}$ be the set of global variables of $P$. Let $2 K-1$ be the bound on the number of context switches.

The result of our reduction is a sequential program $P^{s}$. It has three parts, performed in sequence: the first part $T_{1}^{s}$ is a reduction of $T_{1}$; the second part $T_{2}^{s}$ is a reduction of $T_{2}$; and the third part, Checker, consists of multiple assume statements to verify that a correct interleaving was performed. Let $L_{i}$ be the label preceding the $i^{\text {th }}$ part. $P^{s}$ has the form shown in the first column of Fig. 1

The global variables of $P^{s}$ are $K$ copies of $\operatorname{VAR}_{G}$. If $\operatorname{VAR}_{G}=\left\{\mathrm{x}_{1}, \cdots, \mathrm{x}_{n}\right\}$, then let $\operatorname{VAR}_{G}^{i}=\left\{\mathrm{x}_{1}^{i}, \cdots, \mathrm{x}_{n}^{i}\right\}$. The initial values of $\operatorname{VAR}_{G}^{i}$ are a set of symbolic constants that represent the $i^{\text {th }}$ guess $s_{i}$. $P^{s}$ has an additional global variable $\mathrm{k}$, which will take values between 1 and $K+1$. It tracks the current execution context of a thread: at any time $P^{s}$ can only read and write to variables in $\operatorname{VAR}_{G}^{\mathrm{k}}$. The local variables of $T_{i}^{s}$ are the same as those of $T_{i}$.

Let $\tau(\mathrm{x}, i)=\mathrm{x}^{i}$. If st is a program statement in $P$, let $\tau(\mathrm{st}, i)$ be the statement in which each global variable $\mathrm{x}$ is replaced with $\tau(\mathrm{x}, i)$, and the local variables remain unchanged. The reduction constructs $T_{i}^{s}$ from $T_{i}$ by replacing each statement st by what is shown in the second column of Fig. 11. The third column shows Checker. Variables $\operatorname{VAR}_{G}^{1}$ are initialized to the same values as $\operatorname{VAR}_{G}$ in $P$. Variable $\mathrm{x}_{j}^{i}$, when $i \neq 1$, is initialized to the symbolic constant $v_{j}^{i}$ (which is later referenced inside Checker), and $\mathrm{k}$ is initialized to 1 .

Because local variables are not replicated, a thread resumes execution from the same local state it was in when it was swapped out at a context switch.

The Checker enforces a correct interleaving of the threads. It checks that the values of global variables when $T_{1}$ starts its $i+1^{\text {st }}$ execution context are the

${ }^{3}$ Such models are often used in model checking and numeric program analysis. 
same as the values produced by $T_{2}$ when $T_{2}$ finished executing its $i^{\text {th }}$ execution context. (Because the execution of $T_{2}^{s}$ happens after $T_{1}^{s}$, each execution context of $T_{2}^{s}$ is guaranteed to use the global state produced by the corresponding execution context of $T_{1}^{s}$.)

The reduction ensures the following property: when $P^{s}$ finishes execution, the variables $\operatorname{VAR}_{G}^{K}$ can have a valuation $s$ if and only if the variables $\operatorname{VAR}_{G}$ in $P$ can have the same valuation after $2 K-1$ context switches.

Symbolic constants. One way to deal with symbolic constants is to consider all possible values for them (eager computation). We show instances of this strategy for Boolean programs (\$3) and for PDSs (\$4). Another way is to lazily consider the set of values they may actually take during the (abstract) execution of the concurrent program, i.e., only consider those values that pass the Checker. We show an instance of this strategy for Boolean programs (\$5).

Multiple threads. If there are $n$ threads, $n>2$, then a precise reasoning for $K$ context switches would require one to consider all possible thread schedulings, e.g., $\left(T_{1} ; T_{2} ; T_{1} ; T_{3}\right),\left(T_{1} ; T_{3} ; T_{2} ; T_{3}\right)$, etc. There are $\mathcal{O}\left((n-1)^{K}\right)$ such schedulings. Previous analyses [16 10,3] enumerate explicitly all these schedulings, and thus have $\mathcal{O}\left((n-1)^{K}\right)$ complexity even in the best case. We avoid this exponential factor as follows: we only consider the round-robin thread schedule $T_{1} ; T_{2} ; \cdots T_{n} ; T_{1} ; T_{2} ; \cdots$ for CBA, and bound the length of this schedule instead of bounding the number of context switches. Because a thread is allowed to perform no steps during its execution context, CBA still considers other schedules. For example, when $n=3$, the schedule $T_{1} ; T_{2} ; T_{1} ; T_{3}$ will be considered by CBA only when $K=5$ (in the round-robin schedule, $T_{3}$ does nothing in its first execution context, and $T_{2}$ does nothing in its second execution context).

Setting the bound on the length of the round-robin schedule to $n K$ allows CBA to consider all thread schedulings with $K$ context switches (as well as some schedulings with more than $K$ context switches). Under such a bound, a schedule has $K$ execution contexts per thread. The reduction for multiple threads proceeds in a similar way to the reduction for two threads. The global variables are copied $K$ times. Each thread $T_{i}$ is transformed to $T_{i}^{s}$, as shown in Fig. 1, and $P^{s}$ calls the $T_{i}^{s}$ in sequence, followed by Checker. Checker remains the same (it only has to check that the state after the execution of $T_{n}^{s}$ agrees with the symbolic constants).

The advantages of this approach are as follows: $(i)$ we avoid an explicit enumeration of $\mathcal{O}\left((n-1)^{K}\right)$ thread schedules, thus, allowing our analysis to be more efficient in the common case; $(i i)$ we explore more of the program behavior with a round-robin bound of $n K$ than with a context-switch bound of $K$; and (iii) the cost of analyzing the round-robin schedule of length $n K$ is about the same (in fact, better) than what previous analyses take for exploring one schedule with a context bound of $K$ (see \$4). These advantages allow our analysis to scale much better in the presence of multiple threads than previous analyses. 
In the rest of the paper, we only consider two threads because the extension to multiple threads is straightforward for round-robin scheduling.

Applicability of the reduction to different analyses. Certain analysis, like affine-relation analysis (ARA) over integers, as developed in [11, cannot make use of this reduction. The presence of assume statements makes the ARA problem undecidable. However, any abstraction prepared to deal with branching conditions can also handle assume statements.

It is harder to make a general claim as to whether most sequential analyses can handle symbolic values. One place where symbolic values are used in sequential analyses is to construct summaries for recursive procedures. Eager computation of a procedure summary is similar to analyzing the procedure while assuming symbolic values for the parameters of the procedure.

\section{The Reduction for Boolean Programs}

Boolean Programs. A Boolean program consists of a set of procedures, represented using their control-flow graphs (CFGs). The program has a set of global variables, and each procedure has a set of local variables, where each variable can only receive a Boolean value. Each edge in the CFG is labeled with a statement that can read from and write to variables in scope, or call a procedure. An example is shown in Fig. 2.

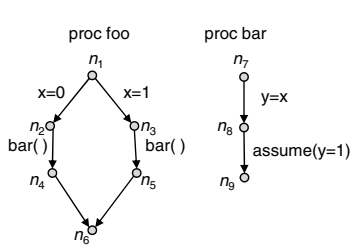

Fig. 2. A Boolean program

For ease of exposition, we assume that all procedures have the same number of local variables, and that they do not have any parameters. Furthermore, the global variables can have any value when program execution starts, and similarly for the local variables when a procedure is invoked.

Let $G$ be the set of valuations of the global variables, and $L$ be the set of valuations of the local variables. A program data-state is an element of $G \times L$. Each program statement st can be associated with a relation $\llbracket \mathrm{st} \rrbracket \subseteq(G \times L) \times(G \times L)$ such

that $\left(g_{0}, l_{0}, g_{1}, l_{1}\right) \in \llbracket \mathrm{st} \rrbracket$ when the execution of st on the state $\left(g_{0}, l_{0}\right)$ can lead to the state $\left(g_{1}, l_{1}\right)$. For instance, in a procedure with one global variable $\mathrm{x}_{1}$ and one local variable $\mathrm{x}_{2}, \llbracket \mathrm{x}_{1}=\mathrm{x}_{2} \rrbracket=\{(a, b, b, b) \mid a, b \in\{0,1\}\}$ and $\llbracket$ assume $\left(\mathrm{x}_{1}=\mathrm{x}_{2}\right) \rrbracket=\{(a, a, a, a) \mid a \in\{0,1\}\}$.

The goal of analyzing such programs is to compute the set of data-states that can reach a program node. This is done using the rules shown in Fig. 3 [1. These rules follow standard interprocedural analyses 1922. Let entry(f) be the entry node of procedure $f, \operatorname{proc}(n)$ the procedure that contains node $n$, ep $(n)=\operatorname{entry}(\operatorname{proc}(n))$, and exitnode $(n)$ is true when $n$ is the exit node of its procedure. Let $\operatorname{Pr}$ be the set of procedures of the program, which includes a distinguished procedure main. The rules of Fig. 3 compute three types of relations: $H_{n}\left(g_{0}, l_{0}, g_{1}, l_{1}\right)$ denotes the fact that if $\left(g_{0}, l_{0}\right)$ is the data state at $\operatorname{entry}(n)$, then the data state $\left(g_{1}, l_{1}\right)$ can reach node $n ; S_{\mathrm{f}}$ is the summary relation 


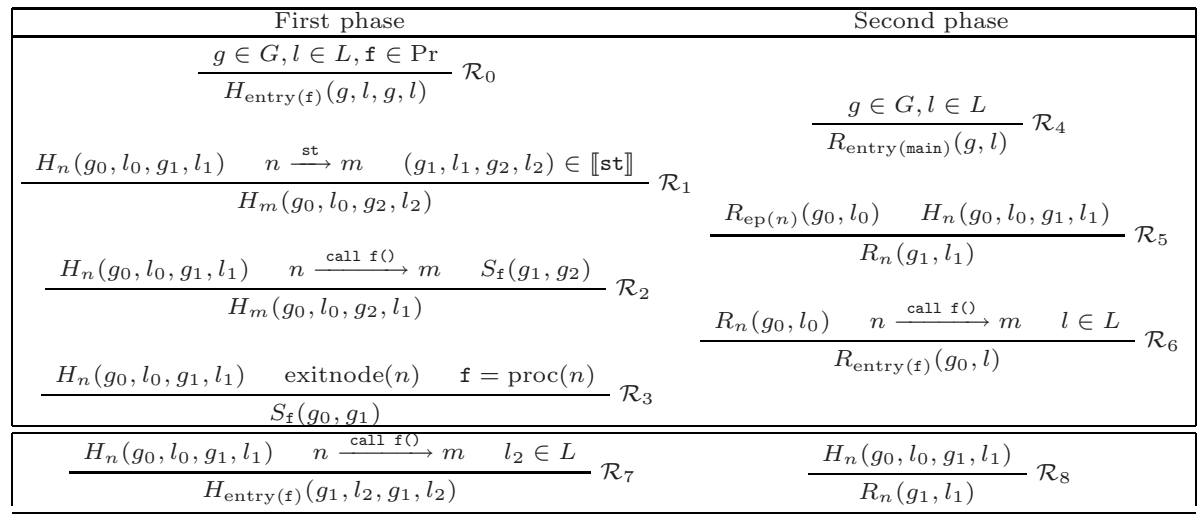

Fig. 3. Rules for the analysis of Boolean programs

for procedure $\mathrm{f}$, which captures the net transformation that an invocation of the procedure can have on the global state; $R_{n}$ is the set of data states that can reach node $n$. All relations are initialized to be empty.

Eager analysis. Rules $\mathcal{R}_{0}$ to $\mathcal{R}_{6}$ describe an eager analysis. The analysis proceeds in two phases. In the first phase, the rules $\mathcal{R}_{0}$ to $\mathcal{R}_{3}$ are used to saturate the relations $H$ and $S$. In the next phase, this information is used to build the relation $R$ using rules $\mathcal{R}_{4}$ to $\mathcal{R}_{6}$.

Lazy analysis. Let rule $\mathcal{R}_{0}^{\prime}$ be the same as $\mathcal{R}_{0}$ but restricted to just the main procedure. Then the rules $\mathcal{R}_{0}^{\prime}, \mathcal{R}_{1}, \mathcal{R}_{2}, \mathcal{R}_{3}, \mathcal{R}_{7}, \mathcal{R}_{8}$ describe a lazy analysis. The rule $\mathcal{R}_{7}$ restricts the analysis of a procedure to only those states it is called in. As a result, the second phase gets simplified and consists of only the rule $\mathcal{R}_{8}$.

Practical implementations [120] use BDDs to encode each of the relations $H, S$, and $R$ and the rule applications are changed into BDD operations. For

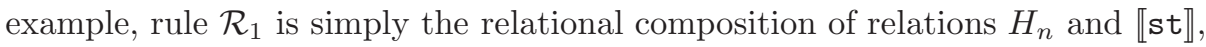
which can be implemented efficiently using BDDs.

Concurrent Boolean Programs. A concurrent Boolean program consists of one Boolean program per thread. The Boolean programs share their set of global variables. In this case, we can apply the reduction presented in 92 to obtain a single Boolean program by making the following changes to the reduction: $(i)$ the variable $\mathrm{k}$ is modeled using a vector of $\log (K)$ Boolean variables, and the increment operation implemented using a simple Boolean circuit on these variables; $(i i)$ the if conditions are modeled using assume statements; and (iii) the symbolic constants are modeled using additional global variables that are not modified in the program. Running any sequential analysis algorithm, and projecting out the values of the $K^{\text {th }}$ set of global variables from $R_{n}$ gives the precise set of reachable global states at node $n$ in the concurrent program. 
The worst-case complexity of analyzing a Boolean program $P$ is bounded by $\mathcal{O}\left(|P||G|^{3}|L|^{2}\right)$, where $|P|$ is the number of program statements. Thus, using our approach, a concurrent Boolean program $P_{c}$ with $n$ threads, and $K$ execution contexts per thread (with round-robin scheduling), can be analyzed in time $\mathcal{O}\left(K\left|P_{c}\right|\left(K|G|^{K}\right)^{3}|L|^{2}|G|^{K}\right)$ : the size of the sequential program obtained from $P_{c}$ is $K\left|P_{c}\right|$; it has the same number of local variables, and its global variables have $K|G|^{K}$ number of valuations. Additionally, the symbolic constants can take $|G|^{K}$ number of valuations, adding an extra multiplicative factor of $|G|^{K}$. The analysis scales linearly with the number of threads $\left(\left|P_{c}\right|\right.$ is $\left.\mathcal{O}(n)\right)$.

This reduction actually applies to any model that works with finite-state data, which includes Boolean programs with references 214. In such models, the heap is assumed to be bounded in size. The heap is included in the global state of the program, hence, our reduction would create multiple copies of the heap, initialized with symbolic values. Our experiments (86) used such models.

Such a process of duplicating the heap can be expensive when the number of heap configurations that actually arise in the concurrent program is very small compared to the total number of heap configurations possible. The lazy version of our algorithm (\$5) addresses this issue.

\section{The Reduction for PDSs}

PDSs are also popular models of programs. The motivation for presenting the reduction for PDSs is that it allows one to apply the numerous algorithms developed for PDSs for CBA. For instance, one can use backward analysis of PDSs to get a backward analysis on concurrent programs.

Definition 1. A pushdown system is a triple $\mathcal{P}=(P, \Gamma, \Delta)$, where $P$ is a set of states, $\Gamma$ is a set of stack symbols, and $\Delta \subseteq P \times \Gamma \times P \times \Gamma^{*}$ is a finite set of rules. A configuration of $\mathcal{P}$ is a pair $\langle p, u\rangle$ where $p \in P$ and $u \in \Gamma^{*}$. A rule $r \in \Delta$ is written as $\langle p, \gamma\rangle \hookrightarrow\left\langle p^{\prime}, u\right\rangle$, where $p, p^{\prime} \in P, \gamma \in \Gamma$ and $u \in \Gamma^{*}$. These rules define a transition relation $\Rightarrow_{\mathcal{P}}$ on configurations of $\mathcal{P}$ as follows: If $r=\langle p, \gamma\rangle \hookrightarrow\left\langle p^{\prime}, u^{\prime}\right\rangle$ then $\left\langle p, \gamma u^{\prime \prime}\right\rangle \Rightarrow_{\mathcal{P}}\left\langle p^{\prime}, u^{\prime} u^{\prime \prime}\right\rangle$ for all $u^{\prime \prime} \in \Gamma^{*}$. The reflexive transitive closure of $\Rightarrow_{\mathcal{P}}$ is denoted by $\Rightarrow_{\mathcal{P}}^{*}$.

Without loss of generality, we restrict the PDS rules to have at most two stack symbols on the right-hand side [21.

The standard way of modeling control-flow of programs using PDSs is as follows: the set $P$ consists of a single state $\{p\}$; the set $\Gamma$ consists of program nodes, and $\Delta$ has one rule per edge in the control-flow graph as follows: $\langle p, u\rangle \hookrightarrow$ $\langle p, v\rangle$ for an intraprocedural edge $(u, v) ;\langle p, u\rangle \hookrightarrow\langle p, e v\rangle$ for a procedure call at node $u$ that returns to $v$ and calls the procedure starting at $e ;\langle p, u\rangle \hookrightarrow\langle p, \varepsilon\rangle$ if $u$ is the exit node of a procedure. Finite-state data is encoded by expanding $P$ to be the set of global states, and expanding $\Gamma$ by including valuations of local variables. Under such an encoding, a configuration $\left\langle p, \gamma_{1} \gamma_{2} \cdots \gamma_{n}\right\rangle$ represents the instantaneous state of the program: $p$ is the valuation of global variables, $\gamma_{1}$ has 


\begin{tabular}{|c|}
\hline $\begin{array}{c}\text { For each }\langle p, \gamma\rangle \hookrightarrow\left\langle p^{\prime}, u\right\rangle \in\left(\Delta_{1} \cup \Delta_{2}\right) \text { and for all } p_{i} \in P, k \in\{1, \cdots, K\}: \\
\left\langle\left(k, p_{1}, \cdots, p_{k-1}, p, p_{k+1}, \cdots, p_{K}\right), \gamma\right\rangle \hookrightarrow\left\langle\left(k, p_{1}, \cdots, p_{k-1}, p^{\prime}, p_{k+1}, \cdots, p_{K}\right), u\right\rangle\end{array}$ \\
\hline \hline For each $\gamma \in \Gamma_{j}$ and for all $p_{i} \in P, k \in\{1, \cdots, K\}:$ \\
$\left\langle\left(k, p_{1}, \cdots, p_{K}\right), \gamma\right\rangle \hookrightarrow\left\langle\left(k+1, p_{1}, \cdots, p_{K}\right), \gamma\right\rangle$ \\
$\left\langle\left(K+1, p_{1}, \cdots, p_{K}\right), \gamma\right\rangle \hookrightarrow\left\langle\left(1, p_{1}, \cdots, p_{K}\right), e_{j+1} \gamma\right\rangle$ \\
\hline
\end{tabular}

Fig. 4. PDS rules for $\mathcal{P}_{s}$

the current program location and values of local variables in scope, and $\gamma_{2} \cdots \gamma_{n}$ store the return addresses and values of local variables for unfinished calls.

A concurrent program with two threads is represented with two PDSs that share their global state: $\mathcal{P}_{1}=\left(P, \Gamma_{1}, \Delta_{1}\right), \mathcal{P}_{2}=\left(P, \Gamma_{2}, \Delta_{2}\right)$. A configuration of such a system is the triplet $\left\langle p, u_{1}, u_{2}\right\rangle$ where $p \in P, u_{1} \in \Gamma_{1}^{*}, u_{2} \in \Gamma_{2}^{*}$. Define two transition systems: if $\left\langle p, u_{i}\right\rangle \Rightarrow_{\mathcal{P}_{i}}\left\langle p^{\prime}, u_{i}^{\prime}\right\rangle$ then $\left\langle p, u_{1}, u\right\rangle \Rightarrow_{1}\left\langle p^{\prime}, u_{1}^{\prime}, u\right\rangle$ and $\left\langle p, u, u_{2}\right\rangle \Rightarrow_{2}\left\langle p^{\prime}, u, u_{2}^{\prime}\right\rangle$ for all $u$. The problem of interest with concurrent programs, under a context bound $2 K-1$, is to find the reachable states under the transition system $\left(\Rightarrow_{1}^{*} ; \Rightarrow_{2}^{*}\right)^{K}$ (here the semicolon denotes relational composition, and exponentiation is repeated relational composition).

We reduce the concurrent program $\left(\mathcal{P}_{1}, \mathcal{P}_{2}\right)$ to a single $\operatorname{PDS} \mathcal{P}_{s}=\left(P_{s}, \Gamma_{s}, \Delta_{s}\right)$. Let $P_{s}$ be the set of all $K+1$ tuples whose first component is a number between 1 and $K$, and the rest are from the set $P$, i.e., $P_{s}=\{1, \cdots, K\} \times P \times P \times \cdots \times P$. This set relates to the reduction from $₫ 2$ as follows: an element $\left(k, p_{1}, \cdots, p_{K}\right) \in$ $P_{s}$ represents that the value of the variable $\mathrm{k}$ is $k$; and $p_{i}$ encodes a valuation of the variables $\operatorname{VAR}_{G}^{i}$. When $\mathcal{P}_{s}$ is in such a state, its rules would only modify $p_{k}$.

Let $e_{i} \in \Gamma_{i}$ be the starting node of the $i^{\text {th }}$ thread. Let $\Gamma_{s}$ be the disjoint union of $\Gamma_{1}, \Gamma_{2}$ and an additional symbol $\left\{e_{3}\right\} . \mathcal{P}_{s}$ does not have an explicit checking phase. The rules $\Delta_{s}$ are defined in Fig. 4 .

We deviate slightly from the reduction presented in $\$ 2$ by changing the goto statement, which passes control from the first thread to the second, into a procedure call. This ensures that the stack of the first thread is left intact when control is passed to the next thread. Furthermore, we assume that the PDSs cannot empty their stacks, i.e., it is not possible that $\left\langle p, e_{1}\right\rangle \Rightarrow_{\mathcal{P}_{1}}^{*}\left\langle p^{\prime}, \varepsilon\right\rangle$ or $\left\langle p, e_{2}\right\rangle \Rightarrow_{\mathcal{P}_{2}}^{*}\left\langle p^{\prime}, \varepsilon\right\rangle$ for all $p, p^{\prime} \in P$ (in other words, the main procedure should not return). This can be enforced for arbitrary PDSs [8].

Theorem 1. Starting execution of the concurrent program $\left(\mathcal{P}_{1}, \mathcal{P}_{2}\right)$ from the state $\left\langle p, e_{1}, e_{2}\right\rangle$ can lead to the state $\left\langle p^{\prime}, c_{1}, c_{2}\right\rangle$ under the transition system $\left(\Rightarrow_{1}^{*} ; \Rightarrow_{2}^{*}\right)^{K}$ if and only if there exist states $p_{2}, \cdots, p_{K} \in P$ such that $\left\langle\left(1, p, p_{2}, \cdots, p_{K}\right), e_{1}\right\rangle \Rightarrow_{\mathcal{P}_{s}}\left\langle\left(1, p_{2}, p_{3}, \cdots, p_{K}, p^{\prime}\right), e_{3} c_{2} c_{1}\right\rangle$.

Note that the checking phase is implicit in the statement of Thm. 1

Complexity. Using our reduction, one can find the set of all reachable configurations of the concurrent program $\left(\mathcal{P}_{1}, \mathcal{P}_{2}\right)$ in time $\mathcal{O}\left(K^{2}|P|^{2 K}\left|\operatorname{Proc} \| \Delta_{1}+\Delta_{2}\right|\right)$, 
where $\mid$ Proc $\mid$ is the number of procedures in the progrant 4 ]. Using backward reachability algorithms, one can verify if a given configuration in reachable in time $\mathcal{O}\left(K^{3}|P|^{2 K}\left|\Delta_{1}+\Delta_{2}\right|\right)$. Both these complexities are asymptotically better than those of previous algorithms for PDSs 16 10, with the latter being linear in the program size $\left|\Delta_{1}+\Delta_{2}\right|$.

A similar reduction works for multiple threads as well (under round-robin scheduling). Moreover, the complexity of finding all reachable states under a bound of $n K$ with $n$ threads, using a standard PDS reachability algorithm, is $\mathcal{O}\left(K^{3}|P|^{4 K}|\operatorname{Proc} \| \Delta|\right)$, where $|\Delta|=\Sigma_{i=1}^{n}\left|\Delta_{i}\right|$ is the total number of rules in the concurrent program.

This reduction produces a large number of rules in $\mathcal{P}_{s}$, but we can leverage work on symbolic PDSs 21 to obtain symbolic implementations 8].

\section{Lazy CBA of Concurrent Boolean Programs}

In the reduction presented in $₫ 3$, the analysis of the generated sequential program had to assume all possible values for the symbolic constants. The lazy analysis will have the property that at any time, if the analysis considers the $K$-tuple $\left(g_{1}, \cdots, g_{K}\right)$ of valuations of the symbolic constants, then there is a single valid execution of the concurrent program in which the global state is $g_{i}$ at the end of the $i^{\text {th }}$ execution context of the first thread for all $1 \leq i \leq K$.

The idea is to iteratively build up the effect that each thread can have on the global state in their $K$ execution contexts. Note that $T_{1}^{s}\left(\right.$ or $T_{2}^{s}$ ) does not need to know the values of $\operatorname{VAR}_{G}^{i}$ when $\mathrm{k}<i$. Hence, the analysis proceeds by making no assumptions on the values of $\operatorname{VAR}_{G}^{i}$ when $i>\mathrm{k}$. When $\mathrm{k}$ is incremented to $k+1$ in the analysis of $T_{1}^{s}$, it consults a table $E^{2}$ that stores the effect that $T_{2}^{s}$ can have in its first $k$ execution contexts. Using that table, it figures out a valuation of $\operatorname{VAR}_{G}^{k+1}$ to continue the analysis of $T_{1}^{s}$, and stores the effect that $T_{1}^{s}$ can have in its first $k$ execution contexts in table $E^{1}$. These tables are built iteratively. More technically, if the analysis can deduce that $T_{1}^{s}$, when started in state $\left(1, g_{1}, \cdots, g_{k}\right)$, can reach the state $\left(k, g_{1}^{\prime}, \cdots, g_{k}^{\prime}\right)$, and $T_{2}^{s}$, when started in state $\left(1, g_{1}^{\prime}, \cdots, g_{k}^{\prime}\right)$ can reach $\left(k, g_{2}, g_{3}, \cdots, g_{k}, g_{k+1}\right)$, then an increment of $\mathrm{k}$ in $T_{1}^{s}$ produces the global state $s=\left(k+1, g_{1}^{\prime}, \cdots, g_{k}^{\prime}, g_{k+1}\right)$. Moreover, $s$ can be reached when $T_{1}^{s}$ is started in state $\left(1, g_{1}, \cdots, g_{k+1}\right)$ because $T_{1}^{s}$ could not have touched $\operatorname{VAR}_{G}^{k+1}$ before the increment that changed k to $k+1$. The algorithm is shown in Fig. 5. The entities used in it have the following meanings:

- Let $\bar{G}=\cup_{i=1}^{K} G^{i}$, where $G$ is the set of global states. An element from the set $\bar{G}$ is written as $\bar{g}$. Let $L$ be the set of local states.

- The relation $H_{n}^{j}$ is related to program node $n$ of the $j^{\text {th }}$ thread. It is a subset of $\{1, \cdots, K\} \times \bar{G} \times \bar{G} \times L \times \bar{G} \times L$. If $H_{n}^{j}\left(k, \overline{g_{0}}, \overline{g_{1}}, l_{1}, \overline{g_{2}}, l_{2}\right)$ holds, then each of the $\overline{g_{i}}$ are an element of $G^{k}$ (i.e., a $k$-tuple of global states), and the thread $T_{j}$ is in its $k^{\text {th }}$ execution context. Moreover, if the valuation of

${ }^{4}$ The number of procedures of a PDS is defined as the number of symbols appearing as the first of the two stack symbols on the right-hand side of a call rule. 
$\operatorname{VAR}_{G}^{i}, 1 \leq i \leq k$, was $\overline{g_{0}}$ when $T_{j}^{s}$ (the reduction of $T_{j}$ ) started executing, and if the node $\operatorname{ep}(n)$ could be reached in data state $\left(\overline{g_{1}}, l_{1}\right)$, then $n$ can be reached in data state $\left(\overline{g_{2}}, l_{2}\right)$, and the variables $\operatorname{VAR}_{G}^{i}, i>k$ are not touched (hence, there is no need to know their values).

- The relation $S_{\mathrm{f}}$ captures the summary of procedure $\mathrm{f}$.

- The relations $E^{j}$ store the effect of executing a thread. If $E^{j}\left(k, \overline{g_{0}}, \overline{g_{1}}\right)$ holds, then $\overline{g_{0}}, \overline{g_{1}} \in G^{k}$, and the execution of thread $T_{j}^{s}$, starting from $\overline{g_{0}}$ can lead to $\overline{g_{1}}$, without touching variables in $\operatorname{VAR}_{G}^{i}, i>k$.

- The function $\operatorname{check}\left(k,\left(g_{1}, \cdots, g_{k}\right),\left(g_{1}^{\prime}, \cdots, g_{k}^{\prime}\right)\right)$ returns $g_{k}^{\prime}$ if $g_{i+1}=g_{i}^{\prime}$ for $1 \leq i \leq k-1$, and is undefined otherwise. This function checks for the correct transfer of the global state from $T_{2}$ to $T_{1}$ at a context switch.

- Let $\left[\left(g_{1}, \cdots, g_{i}\right),\left(g_{i+1}, \cdots g_{j}\right)\right]=\left(g_{1}, \cdots, g_{j}\right)$. We sometimes write $g$ to mean $(g)$, i.e., $\left[\left(g_{1}, \cdots, g_{i}\right), g\right]=\left(g_{1}, \cdots, g_{i}, g\right)$.

Understanding the rules. The rules $\mathcal{R}_{1}^{\prime}, \mathcal{R}_{2}^{\prime}, \mathcal{R}_{3}^{\prime}$, and $\mathcal{R}_{7}^{\prime}$ describe intrathread computation, and are similar to the corresponding unprimed rules in Fig. 3. The rule $\mathcal{R}_{10}$ initializes the variables for the first execution context of $T_{1}$. The rule $\mathcal{R}_{12}$ initializes the variables for the first execution context of $T_{2}$. The rules $\mathcal{R}_{8}$ and $\mathcal{R}_{9}$ ensure proper hand off of the global state from one thread to another. These two are the only rules that change the value of $k$. For example, consider rule $\mathcal{R}_{8}$. It ensures that the global state at the end of $k^{\text {th }}$ execution context of $T_{2}$ is passed to the $(k+1)^{\text {th }}$ execution context of $T_{1}$, using the function check. The value $g$ returned by this function represents a reachable valuation of the global variables when $T_{1}$ starts its $(k+1)^{\text {th }}$ execution context.

The following theorem shows that the relations $E^{1}$ and $E^{2}$ are built lazily, i.e., they only contain relevant information. A proof can be found in 8$]$.

Theorem 2. After running the algorithm described in Fig. 5, $E^{1}\left(k,\left(g_{1}, \cdots\right.\right.$, $\left.\left.g_{k}\right),\left(g_{1}^{\prime}, \cdots, g_{k}^{\prime}\right)\right)$ and $E^{2}\left(k,\left(g_{1}^{\prime}, \cdots, g_{k}^{\prime}\right),\left(g_{2}, \cdots, g_{k}, g\right)\right)$ hold if and only if there is an execution of the concurrent program with $2 k-1$ context switches that starts in state $g_{1}$ and ends in state $g$, and the global state is $g_{i}$ at the start of the $i^{\text {th }}$ execution context of $T_{1}$ and $g_{i}^{\prime}$ at the start of the $i^{\text {th }}$ execution context of $T_{2}$. The set of reachable global states of the program in $2 K-1$ context switches are all $g \in G$ such that $E^{2}\left(K, \overline{g_{1}},\left[\overline{g_{2}}, g\right]\right)$ holds.

\section{Experiments}

We did a proof-of-concept implementation of the eager algorithm for Boolean programs, presented in \$3, using the model checker MOPED [20]. We took sequential programs and assumed that there were two copies of the program running concurrently (except for BlueT). The input programs are obtained from a variety of sources: BlueT is a model of a Bluetooth driver [17; Java* are the result of abstracting Java programs [2]; Reg* are from the regression suite of MOPED; Toy is a toy program we wrote for checking correctness. Some programs, especially ones obtained from Java programs, have pointers and a bounded heap (which is 


$$
\begin{aligned}
& \frac{H_{n}^{j}\left(k, \overline{g_{0}}, \overline{g_{1}}, l_{1},\left[\overline{g_{2}}, g_{3}\right], l_{3}\right) \quad n \stackrel{\text { st }}{\longrightarrow} m \quad\left(g_{3}, l_{3}, g_{4}, l_{4}\right) \in \llbracket \text { st } \rrbracket}{H_{m}^{j}\left(k, \overline{g_{0}}, \overline{g_{1}}, l_{1},\left[\overline{g_{2}}, g_{4}\right], l_{4}\right)} \mathcal{R}_{1}^{\prime} \\
& \frac{H_{n}^{j}\left(k, \overline{g_{0}}, \overline{g_{1}}, l_{1}, \overline{g_{2}}, l_{2}\right) \quad n \stackrel{\text { call } \mathrm{f}()}{\longrightarrow} m \quad S_{\mathrm{f}}\left(k+i,\left[\overline{g_{2}}, \bar{g}\right],\left[\overline{g_{3}}, \overline{g^{\prime}}\right]\right)}{H_{m}^{j}\left(k+i,\left[\overline{g_{0}}, \bar{g}\right],\left[\overline{g_{1}}, \bar{g}\right], l_{1},\left[\overline{g_{3}}, \overline{g^{\prime}}\right], l_{2}\right)} \mathcal{R}_{2}^{\prime} \\
& \frac{H_{n}^{j}\left(k, \overline{g_{0}}, \overline{g_{1}}, l_{1}, \overline{g_{2}}, l_{2}\right) \quad \text { exitnode }(n) \quad \mathrm{f}=\operatorname{proc}(n)}{S_{\mathrm{f}}\left(k, \overline{g_{1}}, \overline{g_{2}}\right)} \mathcal{R}_{3}^{\prime} \quad \frac{g \in G, l \in L, e=\operatorname{entry}(\text { main })}{H_{e}^{1}(1, g, g, l, g, l)} \mathcal{R}_{10} \\
& \frac{H_{n}^{j}\left(k, \overline{g_{0}}, \overline{g_{1}}, l_{1}, \overline{g_{2}}, l_{2}\right) \quad n \stackrel{\text { call } \mathrm{f}()}{\longrightarrow} m \quad l_{3} \in L}{H_{\text {entry (f) }}^{j}\left(k, \overline{g_{0}}, \overline{g_{2}}, l_{3}, \overline{g_{2}}, l_{3}\right)} \mathcal{R}_{7}^{\prime} \quad \frac{H_{n}^{j}\left(k, \overline{g_{0}}, \overline{g_{1}}, l_{1}, \overline{g_{2}}, l_{2}\right)}{E^{j}\left(k, \overline{g_{0}}, \overline{g_{2}}\right)} \mathcal{R}_{11} \\
& \frac{H_{n}^{1}\left(k, \overline{g_{0}}, \overline{g_{1}}, l_{1}, \overline{g_{2}}, l_{2}\right) \quad E^{2}\left(k, \overline{g_{2}}, \overline{g_{3}}\right) \quad g=\operatorname{check}\left(\overline{g_{0}}, \overline{g_{3}}\right)}{H_{n}^{1}\left(k+1,\left[\overline{g_{0}}, g\right],\left[\overline{g_{1}}, g\right], l_{1},\left[\overline{g_{2}}, g\right], l_{2}\right)} \mathcal{R}_{8} \quad \frac{E^{1}\left(1, g_{0}, g_{1}\right), l \in L}{H_{e_{2}}^{2}\left(1, g_{1}, g_{1}, l, g_{1}, l\right)} \mathcal{R}_{12} \\
& \frac{H_{n}^{2}\left(k, \overline{g_{0}}, \overline{g_{1}}, l_{1}, \overline{g_{2}}, l_{2}\right) \quad E^{1}\left(k+1,\left[g_{3}, \overline{g_{2}}\right],\left[\overline{g_{0}}, g_{4}\right]\right)}{H_{n}^{2}\left(k+1,\left[\overline{g_{0}}, g_{4}\right],\left[\overline{g_{1}}, g_{4}\right], l_{1},\left[\overline{g_{2}}, g_{4}\right], l_{2}\right)} \mathcal{R}_{9}
\end{aligned}
$$

Fig. 5. Rules for lazy analysis of concurrent Boolean programs

accounted for in the number of variables). We verified if a certain program node was reachable by finding the set of reachable data-states at the node. In most cases, we modified the programs to have both positive and negative instances.

The results are shown in Fig. 6.

\begin{tabular}{|l|c|c|r|r|r|r|}
\hline Prog & Inst & $2 K$ & Time (s) & Prog & \#gvars & \#lvars \\
\hline Toy & pos & 20 & 0.3 & 12 & 5 & 0 \\
Reg-blast1 & neg & 20 & 3.9 & 19 & 7 & 21 \\
Reg-blast1 & pos & 20 & 4.1 & 19 & 7 & 21 \\
Reg-slam1 & pos & 20 & 19.6 & 19 & 1 & 10 \\
BlueT & neg & 20 & 7.2 & 30 & 10 & 1 \\
BlueT & pos & 10 & 7.6 & 30 & 10 & 1 \\
JavaMeeting & neg & 10 & 168.5 & 537 & 16 & 64 \\
JavaMeeting & pos & 10 & 361.3 & 537 & 16 & 64 \\
JavaChange & neg & 10 & 770.8 & 601 & 24 & 38 \\
JavaChange & pos & 10 & 1134.4 & 601 & 24 & 38 \\
\hline
\end{tabular}

Fig. 6. Experiments on finite-datastate models out applying all the required operations. For positive cases this never happens, and all the operations are applied.

\section{Related Work}

Most of the related work on CBA has been covered in the body of the paper. A reduction from concurrent programs to sequential programs was given in [17] for 
the case of two threads and two context switches (it has a restricted extension to multiple threads as well). In such a case, the only thread interleaving is $T_{1} ; T_{2} ; T_{1}$. The context switch from $T_{1}$ to $T_{2}$ is simulated by a procedure call. Then $T_{2}$ is executed on the program stack of $T_{1}$, and at the next context switch, the stack of $T_{2}$ is popped off to resume execution in $T_{1}$. Because the stack of $T_{2}$ is destroyed, the analysis cannot return to $T_{2}$ (hence the context bound of 2 ). Their algorithm cannot be generalized to an arbitrary context bound.

Analysis of message-passing concurrent systems, as opposed to ones having shared memory, has been considered in [4. They bound the number of messages that can be communicated, similar to bound the number of contexts.

There has been a large body of work on verification of concurrent programs. Some recent work is 6 15. However, CBA is different because it allows for precise analysis of complicated program models, including recursion. As future work, it would be interesting to explore CBA with the abstractions used in the aforementioned work.

\section{References}

1. Ball, T., Rajamani, S.: Bebop: A symbolic model checker for Boolean programs. In: SPIN (2000)

2. Berger, F., Schwoon, S., Suwimonteerabuth, D.: jMoped (2005), http://www.informatik.uni-stuttgart.de/fmi/szs/tools/moped/jmoped/

3. Bouajjani, A., Fratani, S., Qadeer, S.: Context-bounded analysis of multithreaded programs with dynamic linked structures. In: Damm, W., Hermanns, H. (eds.) CAV 2007. LNCS, vol. 4590, pp. 207-220. Springer, Heidelberg (2007)

4. Chaki, S., Clarke, E.M., Kidd, N., Reps, T.W., Touili, T.: Verifying concurrent message-passing $\mathrm{C}$ programs with recursive calls. In: Hermanns, H., Palsberg, J. (eds.) TACAS 2006 and ETAPS 2006. LNCS, vol. 3920, pp. 334-349. Springer, Heidelberg (2006)

5. Henzinger, T., Jhala, R., Majumdar, R., Sutre, G.: Lazy abstraction. In: POPL (2002)

6. Henzinger, T.A., Jhala, R., Majumdar, R.: Race checking by context inference. In: PLDI (2004)

7. Knoop, J., Steffen, B.: The interprocedural coincidence theorem. In: CC (1992)

8. Lal, A., Reps, T.: Reducing concurrent analysis under a context bound to sequential analysis. Technical Report 1629, University of Wisconsin (2008)

9. Lal, A., Touili, T., Kidd, N., Reps, T.: Interprocedural analysis of concurrent programs under a context bound. TR-1598, University of Wisconsin (July 2007)

10. Lal, A., Touili, T., Kidd, N., Reps, T.: Interprocedural analysis of concurrent programs under a context bound. In: TACAS (2008)

11. Müller-Olm, M., Seidl, H.: Precise interprocedural analysis through linear algebra. In: POPL (2004)

12. Murphy, B., Lam, M.: Program analysis with partial transfer functions. In: PEPM (2000)

13. Musuvathi, M., Qadeer, S.: Iterative context bounding for systematic testing of multithreaded programs. In: PLDI (2007)

14. Qadeer, S., Rajamani, S.: Deciding assertions in programs with references. Technical Report MSR-TR-2005-08, Microsoft Research, Redmond (January 2005) 
15. Qadeer, S., Rajamani, S.K., Rehof, J.: Summarizing procedures in concurrent programs. In: POPL (2004)

16. Qadeer, S., Rehof, J.: Context-bounded model checking of concurrent software. In: Halbwachs, N., Zuck, L.D. (eds.) TACAS 2005. LNCS, vol. 3440, pp. 93-107. Springer, Heidelberg (2005)

17. Qadeer, S., Wu, D.: KISS: Keep it simple and sequential. In: PLDI (2004)

18. Ramalingam, G.: Context-sensitive synchronization-sensitive analysis is undecidable. In: TOPLAS (2000)

19. Reps, T., Horwitz, S., Sagiv, M.: Precise interprocedural dataflow analysis via graph reachability. In: POPL (1995)

20. Schwoon, S.: Moped, http://www.fmi.uni-stuttgart.de/szs/tools/moped/

21. Schwoon, S.: Model-Checking Pushdown Systems. PhD thesis, Technical Univ. of Munich, Munich, Germany (July 2002)

22. Sharir, M., Pnueli, A.: Two approaches to interprocedural data flow analysis. In: Program Flow Analysis: Theory and Applications, Prentice-Hall, Englewood Cliffs (1981) 